\title{
CONSUMO DE ENERGIA E MACRONUTRIENTES POR ADOLESCENTES DE ESCOLAS PÚBLICAS E PRIVADAS
}

\section{ENERGY AND MACRONUTRIENTS CONSUMPTION BY ADOLESCENTS FROM PUBLIC PRIVATE SCHOOLS}

lleana Mourão KAZAPI

Patrícia Faria Di PIETRO'

Sandra Regina Paulon AVANCINI'

Sérgio Fernando Torres de FREITAS 2

Vera Lúcia Cardoso Garcia TRAMONTE'

\section{RESUMO}

O objetivo deste trabalho foi avaliar a adequação do consumo de energia e macronutrientes em adolescentes de escolas públicas (EPU) e privadas (EPR) do município de Florianópolis, SC. A amostra constou de 797 estudantes: 466 (228 meninas e 238 meninos) de EPU e 331 (175 meninas e 156 meninos) de EPR. Os dados foram obtidos através do método recordatório de 24 horas. Observou-se que aproximadamente $50 \%$ dos adolescentes apresentaram consumo energético insuficiente e mais da metade tiveram um consumo adequado de carboidratos. Entre os adolescentes do sexo masculino que apresentaram alto consumo de carboidratos, houve maior prevalência na rede pública (12,6\% EPU e 3,8\% EPR). Mais da metade dos estudantes apresentaram alto consumo de proteínas (54,3\% EPU e 58,9\% EPR), havendo maior prevalência entre os de sexo masculino (61,7\% masculino e $50,6 \%$ feminino). Apenas $30,5 \%$ dos estudantes das EPU e 36,5\% das EPR apresentaram consumo adequado de lipídios; ressalta-se o elevado percentual de adolescentes com alto consumo deste nutriente (33,9\% EPU e 39,0\% EPR). Entre os estudantes que apresentaram baixo consumo de lipídios, houve maior prevalência dos adolescentes do sexo masculino (35,3\% masculino e 26,8\% feminino), estudantes da EPU (41,6\% EPU e 25,6\% EPR). Pode-se concluir que o padrão alimentar apresentado por estes adolescentes pode estar associado a riscos para a saúde na vida adulta.

Termos de indexação: adolescentes, padrão alimentar, consumo de macronutrientes.

\section{ABSTRACT}

The aim of this study was to evaluate the adequacy of energy and macronutrients consumption in adolescent students from public (EPU) and private schools (EPR) of Florianópolis, state of Santa Catarina. The sample consisted of 797 students: 466 (228 female and 238 male) from public schools and 331 (175 female and 156 male) from private schools. The informations were obtained using the

\footnotetext{
(1) Departamento de Nutrição, Universidade Federal de Santa Catarina. Campus Universitário Trindade, Centro de Ciências da Saúde, Caixa Postal 476, 88040-900, Florianópolis, SC, Brasil. Correspondência para/Correspondence to: I.M.KAZAPI.

(2) Departamento de Saúde Pública, Universidade Federal de Santa Catarina
} 


\begin{abstract}
24-hour recall method. It was observed that around $50 \%$ of the adolescents presented insufficient energy intake and more than $50 \%$ of them had adequate consumption of carbohydrates. The students that had high intake of carbohydrates were male from public schools (12.6\% EPU and 3.8\% EPR). More than $50 \%$ of the students had high protein intake (54.3\% EPU and $58.9 \%$ EPR), with prevalence among boys (61.7\% male and $50.6 \%$ female). Only $30.5 \%$ of the students from public schools and $36.5 \%$ from private schools had adequate lipid consumption, and there was a large pecentage of adolescents with high lipid intake (33.9\% EPU and 39.0\% EPR). Low lipid intake was more prevalent among boys (35.3\% male versus $26.8 \%$ female) and among students from public schools (41.6\% EPU and $25.6 \%$ EPR). It was concluded that the food consumption pattern of those adolescents may be associated with danger to their health in the future.
\end{abstract}

Index terms: adolescents, food pattern, macronutrients consumption.

\section{INTRODUÇÃO}

A Organização Mundial de Saúde, considera como adolescentes a faixa etária compreendida entre 10 a 19 anos (World Health..., 1995). A adolescência se caracteriza por um rápido e intenso crescimento físico e profundas modificações orgânicas e comportamentais. O adolescente parece sempre estar em constante conflito consigo e com o mundo, este comportamento parece refletir no perfil alimentar (Bergada, 1998; Malonay, 1998).

Muitos trabalhos mostram que os adolescentes preferem uma alimentação rápida e monótona (fast food), por serem alimentos bem difundidos entre eles e por serem da "moda". É sabido que estes alimentos apresentam uma alta taxa de gordura e portanto um alto conteúdo energético. A manutenção do peso ideal é uma outra meta que se quer atingir com a população, mas nesta faixa etária, parece ser uma tarefa difícil de ser atingida, porque estudos demonstram que a atividade física diminuiu nas últimas 4 décadas, enquanto as ingestões dietéticas permaneceram inalteradas. Apesar desta preocupação, percebe-se que parte dos adolescentes preocupam-se excessivamente com a estética, realizando dietas desequilibradas, restritas e irregulares (Farthing, 1991; Jonhson et al., 1994; Andersen et al., 1995).

Bull et al. (1992), relatam que desde a década de 70 os hábitos alimentares de adolescentes têm sistematicamente resultado em alta proporção de energia oriunda de gordura. Tem sido constatado que os adolescentes consomem lanches muito semelhantes, e estes lanches apresentam um excesso de energia (Priore, 1998).

Os adolescentes tendem a viver intensamente o dia atual, não se importando como seus hábitos alimentares e ingestão de alimentos possam vir a influenciar sua saúde e estado nutricional (Sargent et al., 1994; Anding et al., 1996). Existem consideráveis diferenças entre o consumo declarado pelos adolescentes e os padrões alimentares recomendados, que se refere aos alimentos e conseqüentemente ao consumo de energia e de nutrientes (Dwyer, 1997).

As necessidades nutricionais dos adolescentes muitas vezes não são atingidas, no que diz respeito a energia, e aos carboidratos, e o consumo de proteínas, lipídeos, ferro, vitamina C apresentam-se com freqüência acima das recomendações. A ingestão de gordura nesta faixa etária é um fator preocupante, porque a maioria dos inquéritos tem demonstrado que eles possuem o costume de ingerir uma quantidade muito elevada deste nutriente (Bergströn et al., 1993; Jonhson et al., 1994; Gambardella, 1996).

O consumo de alimentos e os hábitos alimentares de adolescentes na Nova Zelândia, foram relatados por Worsley et al. (1993), onde encontraram um consumo elevado de gorduras e carboidratos simples.

A ingestão de nutrientes por 481 adolescentes franceses foi verificada por Michaud \& Baudier (1991), os quais encontraram elevado consumo de gorduras, entre $36,9 \%$ a $40,5 \%$ entre os meninos e de $34,2 \%$ a $41 \%$ entre as meninas.

Crawley (1993), verificou que na Grã-Bretanha, o percentual de energia proveniente de lipídios foi de $41,4 \%$ entre adolescentes do sexo masculino e $41,6 \%$ entre os do sexo feminino, nível substancialmente maior que o recomendado. Por outro lado, a ingestão de carboidratos apresentou-se menor que o recomendado.

Este trabalho teve como objetivo avaliar a adequação do consumo de macronutrientes (carboidratos, proteínas e lipídios), por adolescentes de escolas públicas (EPU) e privadas (EPR) do município de Florianópolis, SC.

\section{CASUÍSTICA E MÉTODOS}

\section{Amostragem}

Este estudo foi realizado junto às escolas públicas e privadas de Florianópolis, no período de julho de 1996 a 
julho de 1997. Para facilitar a seleção da amostra, considerou-se em um primeiro momento, as escolas como unidade de amostragem. A seleção da escola foi realizada com base nos seguintes critérios: maior número de alunos matriculados e que oferecia ensino de primeiro e segundo grau. Num segundo momento, foram selecionados por sorteio, um conjunto de estudantes em cada escola escolhida anteriormente.

Selecionou-se 797 estudantes, dos quais 331 $(41,53 \%)$ pertenciam à rede de ensino privada e 466 $(58,47 \%)$ à rede de ensino pública. Do total de adolescentes 403 (50,56\%) eram do sexo feminino e 394 (49,44\%) do sexo masculino. Dos adolescentes da rede privada, 175 $(52,87 \%)$ eram do sexo feminino e $156(47,13 \%)$ eram do sexo masculino. Em relação à rede pública, a amostra foi dividida em 228 (48,93\%) meninas e 238 (51,07\%) meninos todos com idade compreendida entre 10 a 18 anos.

\section{Avaliação do consumo dietético}

As informações sobre consumo dietético de energia e de macronutrientes (carboidratos, proteínas e lipídeos) foram coletados através de um inquérito recordatório de 24 horas e foram analisados com o auxílio do software do Centro de Informática em Saúde da Escola Paulista de Medicina (Escola Paulista..., 1995). Os dias das entrevistas, nunca foram às segundas-feiras, para evitar que o dia alimentar analisado fosse um dia atípico de final de semana.

A adequação da contribuição percentual em energia foi analisada de acordo com o recomendado pela Sociedade Brasileira de Alimentação e Nutrição (SBAN) (Vanucchi et al., 1990). Em relação ao consumo energético, considerou-se como adequado aqueles indivíduos que apresentaram entre $90 \%$ a $110 \%$ de adequação em relação às suas necessidades, abaixo deste percentual classificou-se como consumo baixo e acima consumo alto. Considerou-se com consumo adequado aqueles indivíduos que apresentaram de $10 \%$ a $12 \%$ de proteínas; $20 \%$ a $25 \%$ de lipídios e $60 \%$ a $70 \%$ de carboidratos, abaixo destas faixas, baixa adequação e acima, alta adequação.

\section{Análise estatística}

Os testes estatísticos foram feitos através de programa Epi Info versão 6, onde aplicou-se Teste do qui-quadrado $\left(\chi^{2}\right)$.

Uma análise geral foi realizada comparando dados das redes de ensino dos sexos em relação ao consumo baixo, adequado e alto de energia e de macronutrientes, encontrando-se diferenças estatísticas executou-se a análise entre o somatório do consumo baixo e adequado com o consumo alto e o consumo baixo com o somatório do consumo adequado e alto, para encontrar se a significância estatística está no consumo baixo ou alto entre as escolas públicas e privadas.

\section{RESULTADOS E DISCUSSÃO}

\section{Energia}

Em torno de 50,0\% de adolescentes de ambas as redes apresentaram um consumo abaixo das necessidades energéticas. No entanto $32,1 \%$ dos adolescentes da rede pública e $21,8 \%$ da rede privada apresentaram um consumo energético acima do adequado (Figura 1). Os indivíduos do sexo masculino de ambas as redes demonstraram também um baixo consumo energético $(48,1 \%$ EPU e $46,2 \%$ EPR) (Figura 2). Entre adolescentes do sexo feminino, observou-se que mais de $50,0 \%$ apresentaram um consumo energético abaixo do adequado (53,7\% EPU e $63,4 \%$ EPR); as adolescentes da EPR apresentaram uma menor prevalência de alto consumo energético quando comparadas às adolescentes da EPU, diferenças significativas $(31,2 \%$ EPU e $12,6 \%$ EPR) (Figura 3).

$\mathrm{Na}$ ingestão energética entre os sexos, verificou-se diferenças significativas entre os adolescentes que tinham consumo acima do adequado, os do sexo masculino apresentaram maior prevalência $(32,58 \%)$ do que os do sexo feminino $(23,15 \%)$. O consumo energético abaixo da recomendação, foi mais prevalente entre as adolescentes $(57,88 \%)$ do que os adolescentes $(47,37 \%)$. Diferentemente do encontrado nesta pesquisa, outros estudos não tem demonstrado diferenças de consumo energético entre os sexos (McDowell et al., 1994; Corish \& Hurson, 1997).

$\mathrm{O}$ alto consumo de energia apresentado por alguns adolescentes pode facilitar, com o avançar da idade, o sobrepeso e suas conseqüências. É importante que as necessidades de energia desta fase sejam cumpridas, uma vez que poderá causar prejuízos ao crescimento e ao desenvolvimento nesta fase em que estão bastante acelerados (Jacobson, 1998).

\section{Carboidratos}

Verificou-se que mais de 50,0\% dos estudantes apresentaram consumo adequado de carboidratos $(63,3 \%$ EPU e 58,0\% EPR). Ocorreu uma maior prevalência de adolescentes que consumiam acima do recomendado na rede pública (10,7\% EPU e 3,9\% EPR) e uma maior prevalência para baixa adequação na rede privada $(26,0 \%$ EPU e 38,1\% EPR), diferenças estas significativas (Figura 1). Os indivíduos do sexo masculino tiveram um 


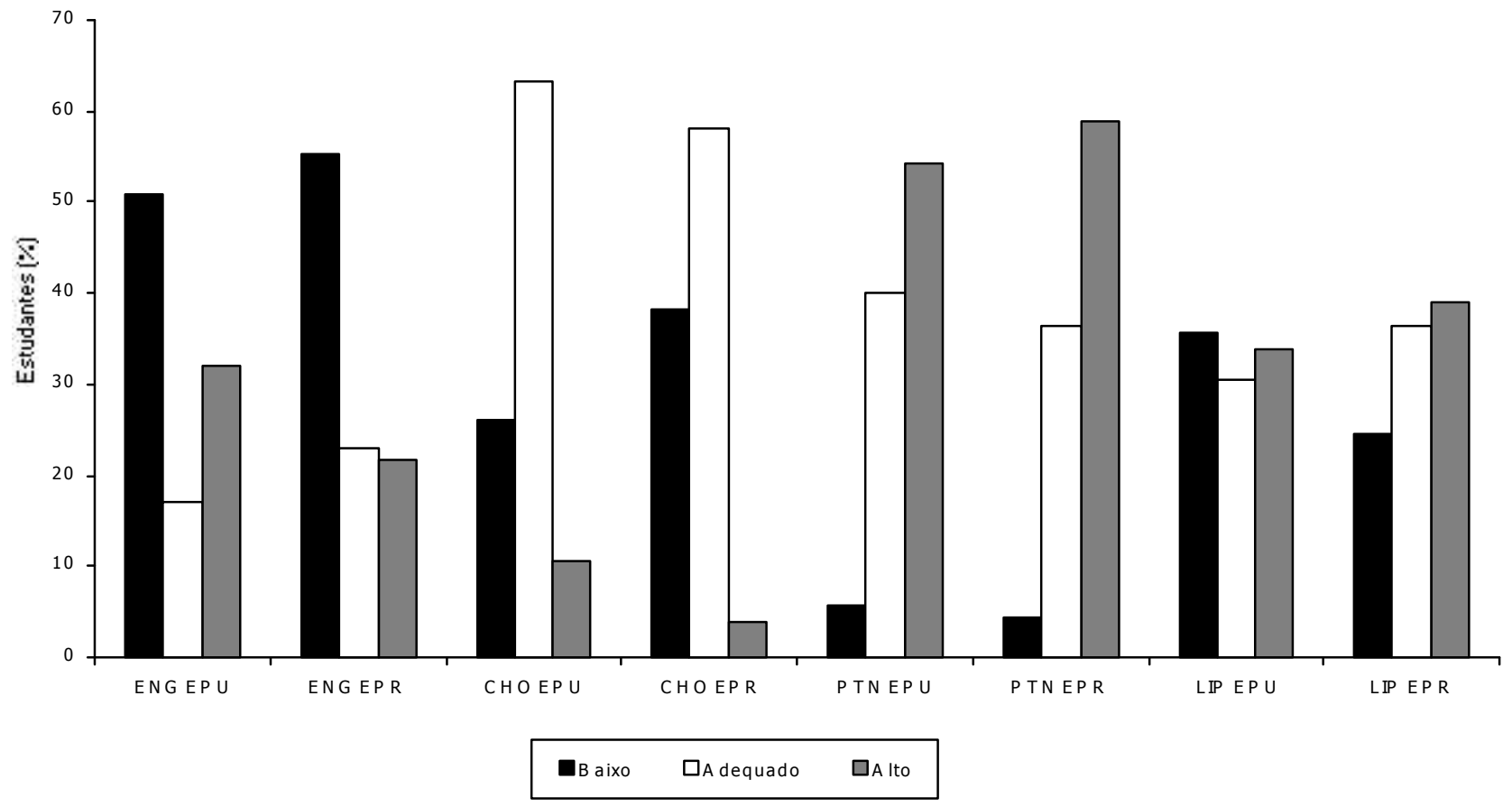

Figura 1. Prevalência da adequação de consumo de energia e macronutrientes de adolescente das redes de ensino de Florianópolis, 1997.

ENG EPU/ ENG EPR: [(baixo+adequado) X (alto)]: $\chi^{2}=10,33(\mathrm{p}<0,01)$.

CHO EPU/CHO EPR: Análise geral: $\chi^{2}=21,36(p<0,0001)$; [(baixo + adequado) X (alto)]: $\chi^{2}=12,30(p<0,001)$; [(baixo) $X$ (adequado + alto)]: $\chi^{2}=13,25(\mathrm{p}<0,001)$.

LIP EPU/ LIP EPR: Análise geral: $\chi^{2}=11,32(p<0,01)$; [(baixo) X (adequado + alto)]: $\chi^{2}=11,25(p<0,001)$.

$\mathrm{ENG}=$ Energia; $\mathrm{CHO}=$ Carboidratos; PTN = Proteina; LIP = Lipídios; EPU = Escolas Públicas; EPR $=$ Escolas Privadas.

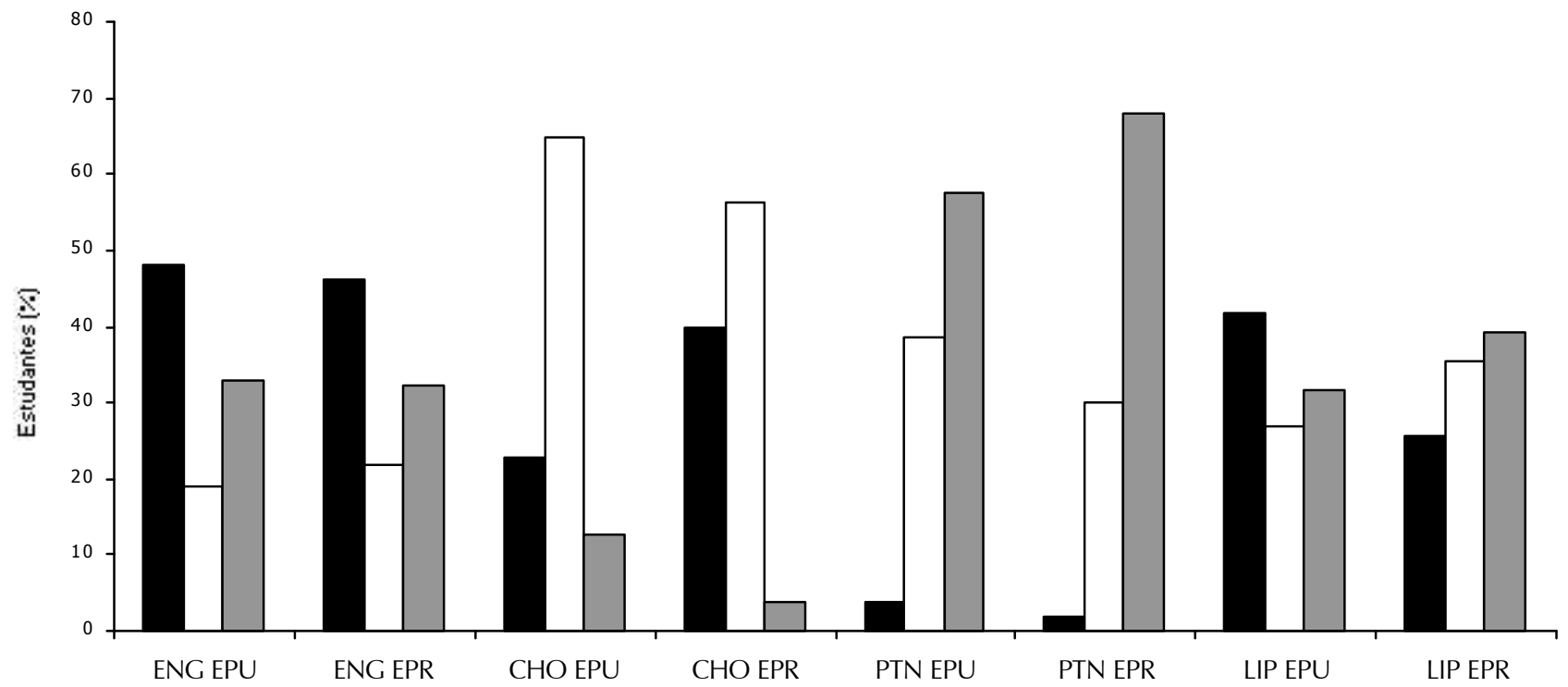

B Baixo $\square$ A dequado $\quad \square$ Ito

Figura 2. Prevalência de adolescentes do sexo masculino quanto a adequação de consumo de energia e macronutrientes das redes de ensino de Florianópolis, 1997.

CHO EPU/ CHO EPR: Análise geral: $\chi^{2}=18,28(p<0,001)$; [(baixo + adequado) X (alto)]: $\chi^{2}=8,71(p<0,01)$; [(baixo) $X$ (adequado + alto)]: $\chi^{2}=13,19(p<0,001)$.

LIP EPU/ LIP EPR: Análise geral: $\chi^{2}=10,56(p<0,01)$; [(baixo) X (adequado + alto)]: $\chi^{2}=10,51(p<0,01)$.

$\mathrm{ENG}=$ Energia; $\mathrm{CHO}=$ Carboidratos; PTN = Proteina; LIP = Lipídios; EPU = Escolas Públicas; EPR = Escolas Privadas. 


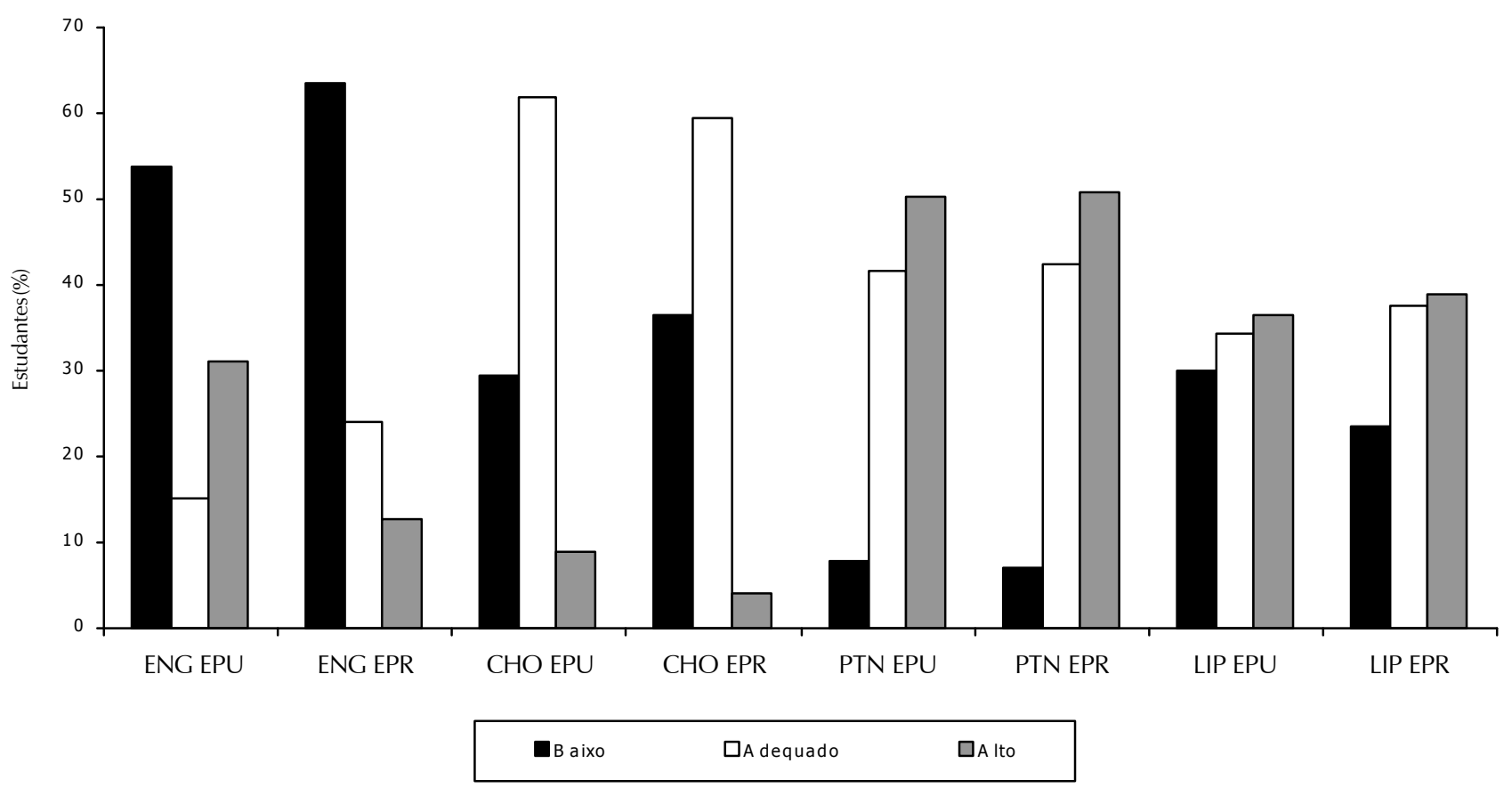

Figura 3. Prevalência de adolescentes do sexo feminino quanto a adequação de consumo de energia e macronutrientes das redes de ensino de Florianópolis, 1997.

ENG EPU/ ENG EPR: Análise geral: $\chi^{2}=20.62(p<0,0001) ;\left[\left(\right.\right.$ baixo + adequado) X (alto)]: $\chi^{2}=19,36(p<0,0001)$. $\mathrm{ENG}=$ Energia; $\mathrm{CHO}=$ Carboidratos; PTN = Proteina; LIP = Lipídios; EPU = Escolas Públicas; EPR = Escolas Privadas.

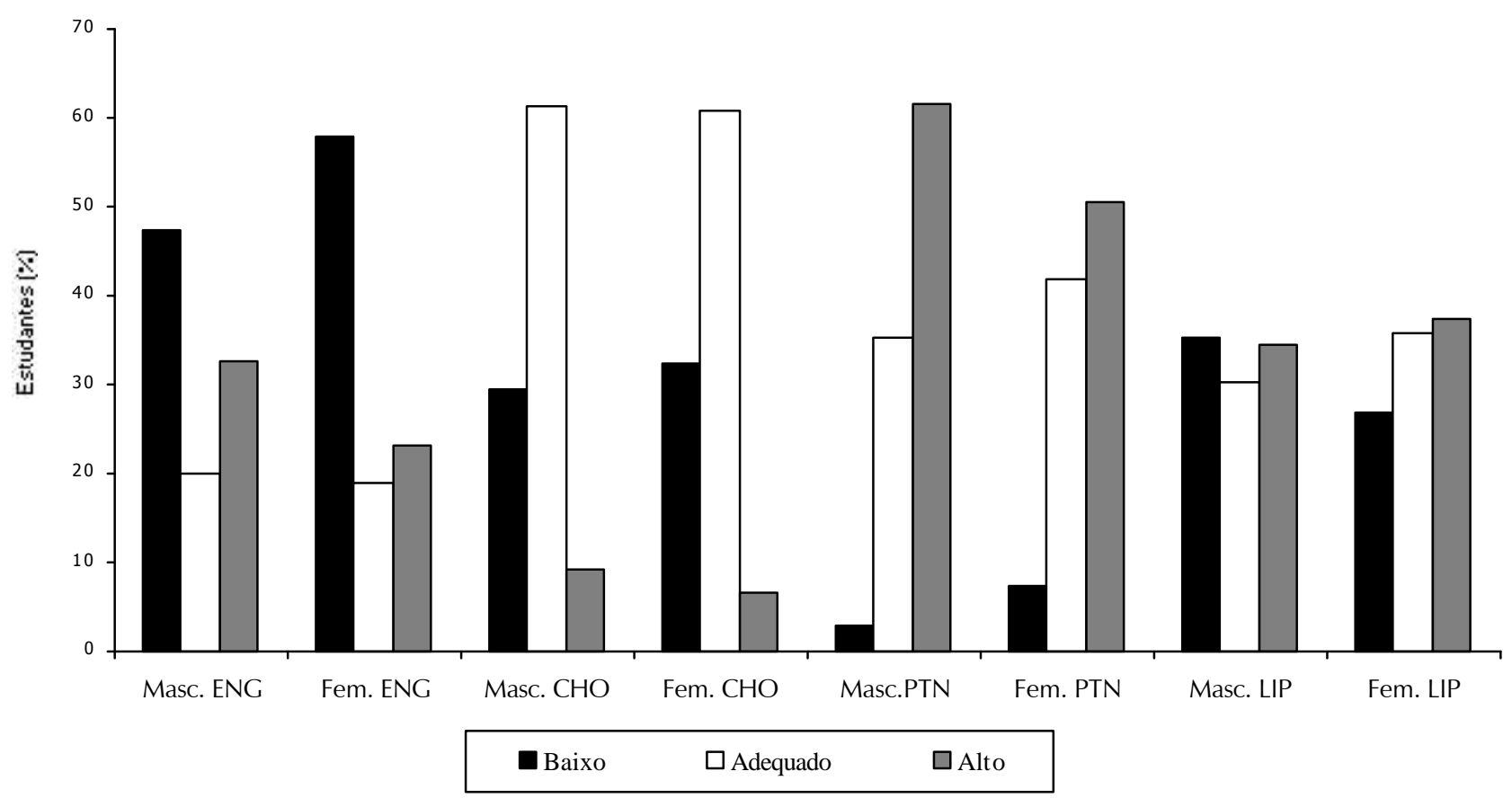

Figura 4. Prevalência de adolescentes de ambos os sexos quanto a adequação de consumo de macronutrientes das redes de ensino de Florianópolis, 1997.

MASC. ENG /FEM. ENG: Análise geral: $\chi^{2}=10,41(p<0,01)$; [(baixo + adequado) X (alto)]: $\chi^{2}=8,91(p<0,01)$; [(baixo) X (adequado + alto)]: $\chi^{2}=8,92(\mathrm{p}<0,01)$.

MASC. PTN / FEM. PTN: Análise geral: $\chi^{2}=13,94(p<0,001)$; [(baixo + adequado) X (alto)]: $\chi^{2}=9,89(p<0,01)$; [(baixo) $X$ (adequado + alto) $]: \chi^{2}=7,72(p<0,01)$.

MASC. LIP /FEM. LIP: Análise geral: $\chi^{2}=6,95(p<0,05)$; [(baixo) X (adequado + alto)]: $\chi^{2}=6,70(p<0,01)$.

$\mathrm{ENG}=$ Energia $; \mathrm{CHO}=$ Carboidratos; PTN $=$ Proteina; LIP = Lipídios; EPU = Escolas Públicas; $\mathrm{EPR}=$ Escolas Privadas. 
comportamento semelhante ao total de adolescentes (Figura 2), enquanto que entre as meninas não houve diferenças significativas entre as redes (Figura 3), apesar de também apresentarem um número elevado de indivíduos com adequação baixa (29,4\% EPU e 36,6\% EPR). Sabe-se que este nutriente é uma importante fonte de energia para o corpo e que se não estiver presente em quantidades adequadas, outros nutrientes desempenharão seu papel, prejudicando o crescimento e desenvolvimento dos indivíduos (Demonte, 1998). Estes resultados são semelhantes aos encontrados por Kazapi \& Ramos (1998), quando estudaram atletas adolescentes de natação.

Quando a comparação é feita entre os sexos (Figura 4), não houve diferenças significativas no consumo de carboidratos. McDowell et al. (1994) também não observaram diferenças significativas no consumo deste macronutriente entre os sexos e ainda verificaram que a ingestão de carboidratos estava entre 50 e $54 \%$ do valor energético total da dieta, menor que a recomendação estabelecida para nossa população (Vannucchi et al., 1990). Verificamos prevalência alta de adolescentes com baixa adequação de consumo de carboidratos (Figuras $1,2,3$ e 4). Sabe-se que nesta faixa etária a atividade física pode estar presente, e que o carboidrato é o principal nutriente para o exercício.

\section{Proteínas}

Ao se analisar a adequação de proteínas, observou-se que mais de $50 \%$ dos adolescentes de ambas as redes de ensino, apresentaram um alto consumo deste nutriente (Figura 1), ocorrendo o mesmo quando analisou-se o consumo protéico entre os do sexo masculino (Figura 2) e do sexo feminino (Figura 3), separadamente. Apesar deste alto consumo protéico, a diferença não foi significativa entre as redes (Figura 1,2,3).

O alto consumo protéico apresentado é um dado relevante, pois este nutriente pode estar sendo desviado de sua função principal, para gerar energia. Além disso, esse alto consumo pode ser explicado, pela falsa crença de que este tipo de nutriente aumenta a força, modela o corpo, aumenta a massa muscular sem a necessidade de praticar exercício físico (Soares et al., 1994). Clavien et al. (1996), encontraram resultados semelhantes a este estudo, onde o consumo protéico foi superior a Recomended Dietary Allowances (RDA). Verificou-se diferenças significativas de consumo protéico entre os sexos (Figura 4). Entre os adolescentes que tinham consumo acima da adequação, os do sexo masculino apresentaram maior prevalência. Foi mais prevalente entre os adolescentes um consumo adequado e abaixo da recomendação. Ao contrário do encontrado neste estudo, as pesquisas tem demonstrado pouca variação no consumo deste nutriente entre os sexos (Crawley, 1993; McDowell et al., 1994; Corish \& Hurson, 1997).

\section{Lipídeos}

Observou-se que 30,5\% dos estudantes das EPU e 36,5\% das EPR apresentaram consumo adequado de lipídios; ressalta-se o elevado percentual de adolescentes com alto consumo deste nutriente (33,9\% EPU e 39,0\% EPR) (Figura 1). Esta alta prevalência também foi observada tanto entre os do sexo masculino (Figura 2) e os do sexo feminino (Figura 3). Dos estudantes que apresentaram baixa adequação de consumo de lipídios, houve maior e significativa prevalência entre os adolescentes da EPU (35,6\% EPU e 24,5\% EPR) (Figura 1) e especificamente entre os do sexo masculino da EPU (41,6\% EPU e 25,6\% EPR) (Figura 2). Não ocorreram diferenças significativas na ingestão lipídica entre as adolescentes das duas redes de ensino (Figura 3). A prevalência de adolescentes que apresentaram baixo consumo lipídico foi maior entre os do sexo masculino (35,3\% masculino e $26,8 \%$ feminino) (Figura 4).

A alta prevalência de consumo de lipídeos pelos adolescentes de ambas as redes de ensino pode favorecer doenças crônicas na vida adulta. Mesmo admitindo que a adolescência é uma fase de experimentação e rebelião contra hábitos alimentares, passa a ser preocupante caso esse padrão alimentar alto em gordura, persistir na idade adulta. Diversas pesquisas mostraram um perfil de consumo alimentar muito semelhante aos dos nossos adolescentes sobretudo de consumo de lipídeos (Michaud \& Baldier, 1991; Bull, 1992; Anderson et al., 1993; Crawley, 1993).

\section{CONCLUSÃO}

Conclui-se que mais da metade dos adolescentes apresentaram um baixo consumo energético, uma adequada ingestão de carboidratos e alta ingestão de proteína, ocorrendo também um elevado número de adolescentes com consumo alto de lipídios. No entanto, estes dados devem ser vistos com cautela uma vez que a análise do consumo percentual de macronutrientes fica comprometida quando o consumo energético é insuficiente sobretudo na análise do consumo protéico. Em relação às diferenças encontradas entre os alunos que freqüentavam escolas da rede pública e privada, observou-se que na rede privada, ocorreu uma menor prevalência de adolescentes do sexo feminino com consumo alto de energia e uma baixa ingestão de carboidratos entre os adolescentes do sexo masculino. Nas escola da rede pública encontrou-se uma maior prevalência de alto consumo de carboidratos e uma baixa ingestão de lipídios entre os adolescentes do sexo masculino. Quando comparou-se as diferenças entre os sexos, os adolescentes do sexo masculino apresentaram uma maior prevalência de alto consumo de energia e proteínas, e baixo consumo de lipídios. 


\section{REFERÊNCIAS BIBLIOGRÁFICAS}

ANDERSEN, L.F., NES, M., SANDSTAD, B., BJORNEBOE, G-E., DREVON, C.A. Dietary intake among Norwegian adolescents. European Journal of Clinical Nutrition, London, v.49, n.8, p.555-564, 1995.

ANDERSON, A.S., MACINTYRE, S., WEST, P. Adolescents meal patterns: grazing habits in the West of Scotland. Health Bull, v.51, n.10, p.158-165, 1993.

ANDING, J.D., KUBENA, K.S., McINTOSH, W.A., O`BRIEN, B. Blood lipids, cardiovascular fitness, obesity, and blod pressure: the presence of potential coronary health disease risk factors in adolescents. Journal of American Dietetic Association, Chicago, v.96, n.3, p.238-242, 1996.

BERGADA, C. A puberdade e a medicina do adolescente. Anais Nestlé, São Paulo, v.55, p.1-8, 1998. Suplemento especial.

BERGSTRÖN, E., HERNELL, O., PERSSON, L.A. Dietary changes in Swedish adolescents. Acta Paediatrica, Oslo, v.82, n.5, p.472-480, 1993.

BULL, N.L. Dietary habits, food consumption, and nutrient intake during adolescence. Journal of Adolescents Health, New York, v.13, n.5, p 384-388, 1992

CLAVIEN, H., THEINTZ, G., RIZZOLI, R. BONJOUR, J.P. Does puberty alter dietary habits in adolescents living in a western society? Journal of Adolescent Health, New York, v.19, n.1, p.68-75, 1996.

CORISH, C., HURSON, M. Evaluation of lifestyle, food consumption and nutrient intake patterns among Irish teenagers. Irish Journal of Medicine Science, v.166, n.4, p.225-230, 1997.

CRAWLEY, H.F. The energy, nutrient and food intakes of teenagers aged 16-17 years in Britain1. Energy, macronutrients and non-starch polysaccharides. British Journal of Nutrition, London, v.70, n.1, p.15-26, 1993.

DEMONTE, A. Carboidratos. In: DUTRA-DE-OLIVEIRA, J.E., MARCHINI, J.S. Ciências Nutricionais. São Paulo : Sarvier, 1998. p.71-85.

DWYER, J.T. Adolescencia. In: CONOCIMIENTOS actuales sobre nutrición. 7.ed. Washington DC : OPS, 1997. p.431441. (Publicación Científica, 565).

ESCOLA PAULISTA DE MEDICINA. Centro de Informática em Saúde. Programa de apoio à nutrição. Versão 2.5. São Paulo, 1995

FARTHING, M.C. Current eating patterns of adolescents in the United States. Nutrition Today, Baltimore, v.26, n.2, p.35-39, 1991.

GAMBARDELLA, A.M.D. Adolescentes, estudantes de período noturno: como se alimentam e gastam suas energias. São
Paulo, 1996. p.42-60. Tese (Doutorado) - Faculdade de Saúde Pública, da Universidade de São Paulo, 1996.

JACOBSON, M.S. Nutrição na adolescência. Anais Nestlé, São Paulo, v.55, p.24-33, 1998. Suplemento especial.

JONHSON, R.K., JONHSON, D.G., WANG, M.Q., SMICIKLAS-WRIGHT, H., GUTHRIE, H.A. Characterizing nutrient intakes of adolescents by sociodemographic factors. Journal of Adolescent Health, New York, v.15, n.2, p.149-154, 1994.

KAZAPI, I.M., RAMOS, L.A.Z. Hábitos e consumo alimentares de atletas nadadores. Revista de Nutrição, Campinas, v.11, n.2, p.117-124, 1998.

MALONAY, M. J. Transtornos do comportamento alimentar na adolescência. Anais Nestlé, São Paulo, v.55, p.18-23, 1998.

McDOWELL, M.A., BRIEFEL, R.R., ALAIMO, K. Energy and macronutrient intake of persons ages 2 months and over in the United States: Third National Health and Nutrition Examination Survey, Phase 1, 1988-1991. Hyattsville : National Center for Health Statistics. 1994. p.1-24. (Advance data from vital health statistics, n. 255$)$.

MICHAUD, C., BAUDIER, F. Habitudes et consummations alimentaires des adolescents français. Synthèse d'enquêtes récents. Archives Françaises Pediatrie, Paris, v.48, n.7, p.475-479, 1991.

PRIORE, S.E. Composição corporal e hábitos alimentares de adolescentes: uma contrição aos indicadores de estado nutricional. São Paulo, 1998. p.51-65. Tese (Doutorado) - Universidade Federal de São Paulo/Escola Paulista de Medicina, 1998.

SARGENT, R.G., KEMPER, K.A., SCHULKEN, E. Dietary behaviors of south Carolina adolescents. Journal of the South Carolina Medical Association, Columbia, v.90, n.6, p.263-269, 1994.

SOARES, E.A., MIDORI, I., BURINI, R.C. Estudo antropométrico e dietético de nadadores competitivos de áreas metropolitanas da região sudeste do Brasil. Revista de Saúde Pública, São Paulo, v.28, n.1, p.9-19, 1994.

VANNUCCHI, H., MENEZES, E.W., CAMPANA, A.O., LAJOLO, F.M. Aplicações das recomendações nutricionais adaptadas à população brasileira. Ribeirão Preto : Regis Suma, 1990. 156p. (Cadernos de Nutrição, 2).

WORLD HEALTH ORGANIZATION. Physical status: the use and interpretation of antropometry. Geneve, 1995. 453p. (Technical Report Series, 854).

WORSLEY, A., WORSLEY, A.J., McCONNON, S., SILVA, P.A. Reported food consumption and dietary habits of New Zealand adolescents. Journal Paediatric Child Health, Carlton, v.29, n.3, p.209-214, 1993.

Recebido para publicação em 21 de junho de 2000 e aceito em 28 de março de 2001. 